\title{
Simultaneous additive and subtractive three-dimensional nanofabrication using integrated two-photon polymerization and multiphoton ablation
}

\author{
Wei Xiong ${ }^{1}$, Yun Shen Zhou $^{1}$, Xiang Nan $\mathrm{He}^{1}$, Yang Gao ${ }^{1}$, Masoud Mahjouri-Samani ${ }^{1}$, Lan Jiang ${ }^{2}$, \\ Tommaso Baldacchini ${ }^{1,3}$ and Yong Feng $\mathrm{Lu}^{1}$
}

Modern three-dimensional nanofabrication requires both additive and subtractive processes. However, both processes are largely isolated and generally regarded as incompatible with each other. In this study, we developed simultaneous additive and subtractive fabrication processes using two-photon polymerization followed by femtosecond (fs) laser multiphoton ablation. To demonstrate the new capability, submicrometer polymer fibers containing periodic holes of $500-\mathrm{nm}$ diameter and microfluidic channels of $1-\mu \mathrm{m}$ diameter were successfully fabricated. This method combining both two-photon polymerization and fs laser ablation improves the nanofabrication efficiency and enables the fabrication of complex three-dimensional micro-/nanostructures, promising for a wide range of applications in integrated optics, microfluidics and microelectromechanical systems.

Light: Science \& Applications (2012) 1, e6; doi:10.1038/Isa.2012.6; published online 13 April 2012

Keywords: femtosecond laser direct writing; femtosecond laser multiphoton ablation; micro-/nanofabrication; two-photon polymerization

\section{INTRODUCTION}

In recent years, three-dimensional (3D) micro-/nanofabrication techniques have attracted increasing attention, due to their promise in a wide range of applications including integrated optics, ${ }^{1,2}$ plasmonics, ${ }^{3,4}$ microbiology $y^{5,6}$ and microelectromechanical systems. ${ }^{7-11}$ Similar to 3D fabrication at macroscopic scales, 3D micro-/nanofabrication generally entails two fundamental approaches: additive and subtractive processes. Among the additive fabrication methods, twophoton polymerization (TPP) is the most predominant. ${ }^{12,13}$ TPP can be simply realized using a tightly focused femtosecond ( $\mathrm{fs}$ ) laser beam to draw 3D nanostructures inside a photocurable polymer. It is by far the only process that allows fabricating nearly arbitrary $3 \mathrm{D}$ structures with a sub-100-nm resolution. Among subtractive fabrication methods, fs laser ablation (FLA) has been applied to a wide variety of materials. ${ }^{14}$ When FLA is applied to transparent materials, structural or phase modification of the materials occurs, leaving behind permanent changes of refractive index or even voids. ${ }^{14,15}$ FLA has found applications in a variety of fields such as optical data storage, ${ }^{16}$ waveguide writing, ${ }^{17}$ and nanosurgery. ${ }^{18}$

Although both additive and subtractive micro-/nanofabrication methods are established, they have been largely isolated and difficult to be integrated, due to either material incompatibilities or significant differences in laser processing parameters for TPP and FLA. Fabricating advanced devices with complex 3D geometries calls for the use of both additive and subtractive processes. For example, a seamless spherical shell is difficult to be fabricated by either additive or subtractive processes alone. In additive processes, the solid shell of the sphere prevents the dissolving of unexposed photoresist inside the sphere to produce a void. While in subtractive processes, a solid sphere has to be created before subtracting the inner part to make a hollow shell. Therefore, a comprehensive 3D micro-/nanofabrication method combining both additive and subtractive functionalities is not only desirable but also in demand for advanced device fabrications.

In this work, we successfully integrated additive (TPP) and subtractive (FLA) processes using a single fs laser direct writing system (The location of Nanoscribe GmbH: Nanoscribe GmbH, Hermannvon-Helmholtz-Platz 1, 76344 Eggenstein-Leopoldshafen, Germany.) in which process conditions could be precisely controlled. The integrated fabrication method not only inherits the merits of both TPP and FLA, such as high resolution beyond the diffraction limit and features with sharp and clean edges, but it also offers the possibility to produce novel device structures which are difficult to be fabricated by either TPP or FLA alone.

\section{MATERIALS AND METHODS}

The whole fabrication process consists of three steps, as shown in Figure 1. First, TPP is employed to fabricate 3D solid microstructures inside a negative photoresist (production name of the liquid 


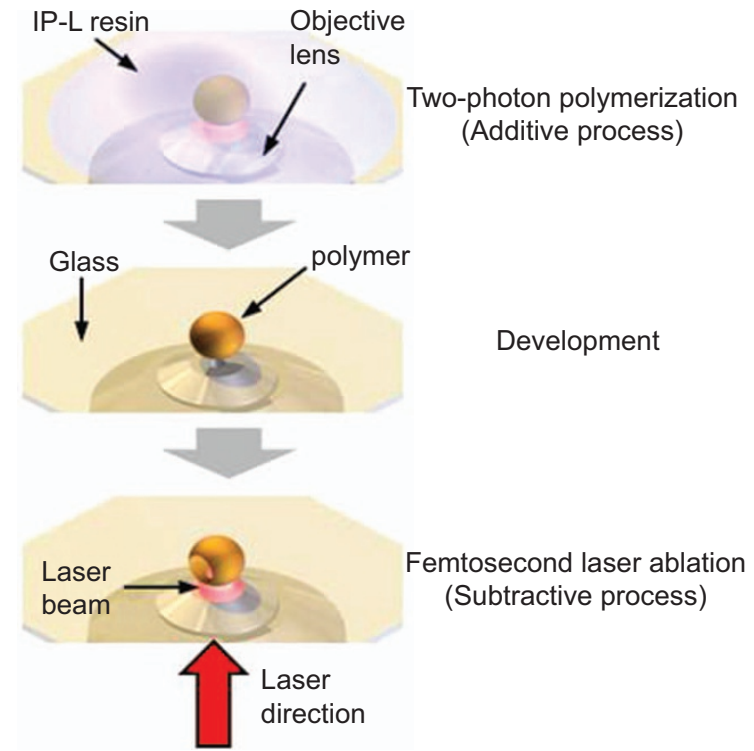

Figure 1 Illustrations of the integrated micro/nanofabrication process.

photoresist formulation with the highest resolution achieved so far from Nanoscribe GmbH (IP-L)). Second, unsolidified photoresist is washed away by rinsing the sample in isopropyl alcohol for $20 \mathrm{~min}$. Third, a subtractive process based on FLA is carried out in air to obtain desired 3D geometries. Depending on the size of the desired structure, TPP was performed using average laser powers that varied between 3.5 and $8 \mathrm{~mW}$. FLA instead, required average laser powers which were 2-7 times higher than those used for TPP. The laser beam was generated by a mode-locked fs fiber laser (780 nm central wavelength, $100 \mathrm{MHz}$ repetition rate, and $120 \mathrm{fs}$ pulse duration). The laser beam was tightly focused within the sample by an oil-immersion objective lens (1.4 NA, $\times 100$ ). Both additive and subtractive fabrications were achieved by moving the sample around a fixed laser beam by a computercontrolled XYZ piezo stage.

\section{RESULTS AND DISCUSSION}

The IP-L photoresist needs to absorb multiple near-infrared photons simultaneously for electron excitation to occur in a single quantum event. Furthermore, the collective energy of the excitation photons should correspond to the near-UV region of the spectrum. ${ }^{12}$ The rate of the multiphoton absorption is proportional to $I^{m}$, where $I$ is the light intensity and $m$ is the number of photons absorbed simultaneously. ${ }^{19}$ Hence, the near-infrared light is strongly absorbed only at the focal point of the laser beam (Figure 2a). The localized absorption of light allows for the confinement of either polymerization in the additive process or ablation in the subtractive process within submicrometer dimensions, beyond the optical diffraction limit of the near-infrared light. In TPP, two near-infrared photons are required to initiate the additive polymerization, ${ }^{12}$ which has a resolution of $100 \mathrm{~nm}$ in this study. In FLA, the number of photons required to induce material removal was determined by studying the absorbance and transmittance spectra of the cured IP-L photoresist (Figure 2b). When completely hardened by UV irradiation, IP-L shows negligible absorption and thus nearly $100 \%$ transmittance at both $780 \mathrm{~nm}$ (corresponding to the energy of one photon) and $390 \mathrm{~nm}$ (corresponding to the collective energy of two photons), respectively, but it has a strong absorption and low transmittance at $260 \mathrm{~nm}$ (corresponding to the collective energy of three photons). This suggests that polymerized IP-L requires the simultaneous absorption of at least three photons to initiate the ablation process when using an fs laser beam with a center wavelength at $780 \mathrm{~nm}$.

Figure 2c shows an array of holes created by FLA in a $200-\mathrm{nm}$ thick film of IP-L that was cured by UV irradiation. A magnified image of one hole is shown in Figure 2d. The diameter of the holes is around $180 \mathrm{~nm}$, far beyond the diffraction limit of the laser beam. In addition, the laser ablation produces holes with sharp and clean edges. Due to the short pulse duration of the fs laser (120 fs) which is much shorter than the electron-phonon coupling time $(\sim 1 \mathrm{ps}),{ }^{20}$ there is limited heat exchange between the irradiated area and the surroundings during the FLA process, resulting in stable and
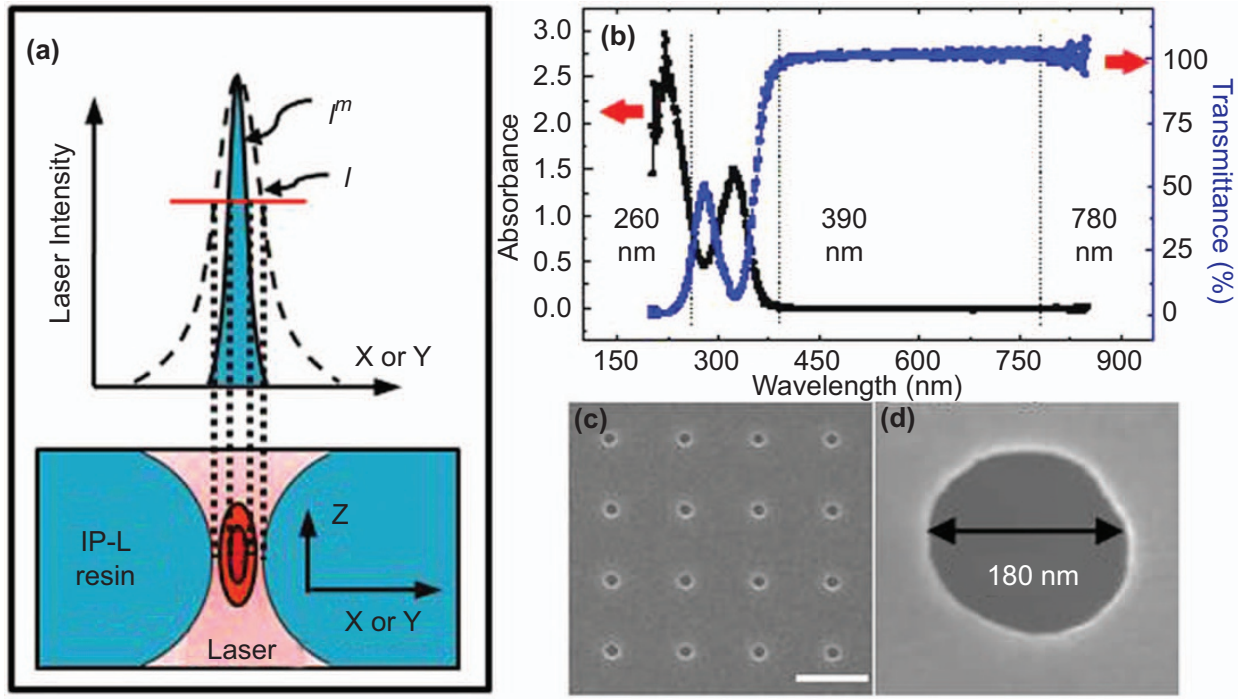

Figure 2 Fundamentals of multiphoton ablation. (a) Schematics of multiphoton absorption; (b) absorbance and transmittance spectra of cured IP-L photoresist; (c) SEM image of the hole-matrix pattern created on a cured IP-L polymer film of 200-nm thickness by fs laser ablation (laser power: $16 \mathrm{~mW}$; exposure time/spot: $0.5 \mathrm{~ms}$ ). The scale bar is $1 \mu \mathrm{m}$; (d) magnified image of a hole. fs, femtosecond; SEM, scanning electron microscope. 

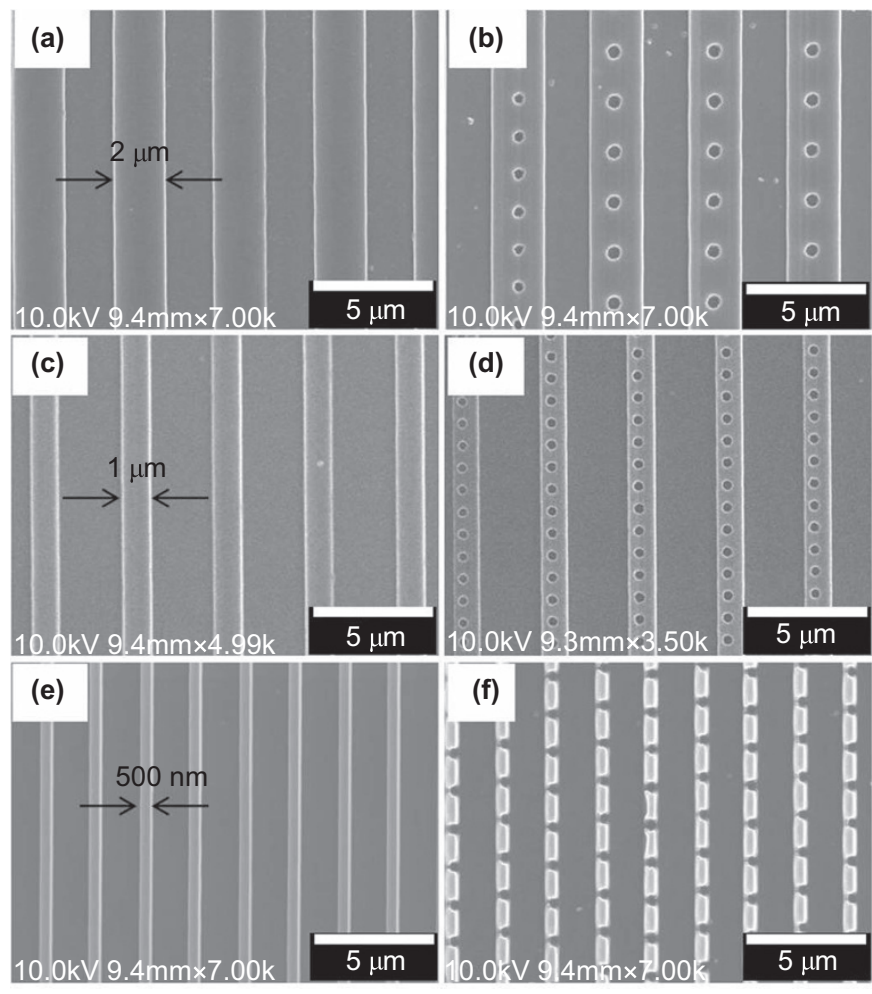

Figure 3 SEM images of polymer fibers fabricated by the 'TPP+FLA' method. (a, c, e) The arrays of fibers created by TPP with 2, 1 and $0.5 \mu \mathrm{m}$ in diameters, respectively; (b, $\mathbf{d}, \mathbf{f})$ the arrays of fibers with periodic hole patterns after the FLA process. FLA, femtosecond laser ablation; SEM, scanning electron microscope; TPP, two-photon polymerization.
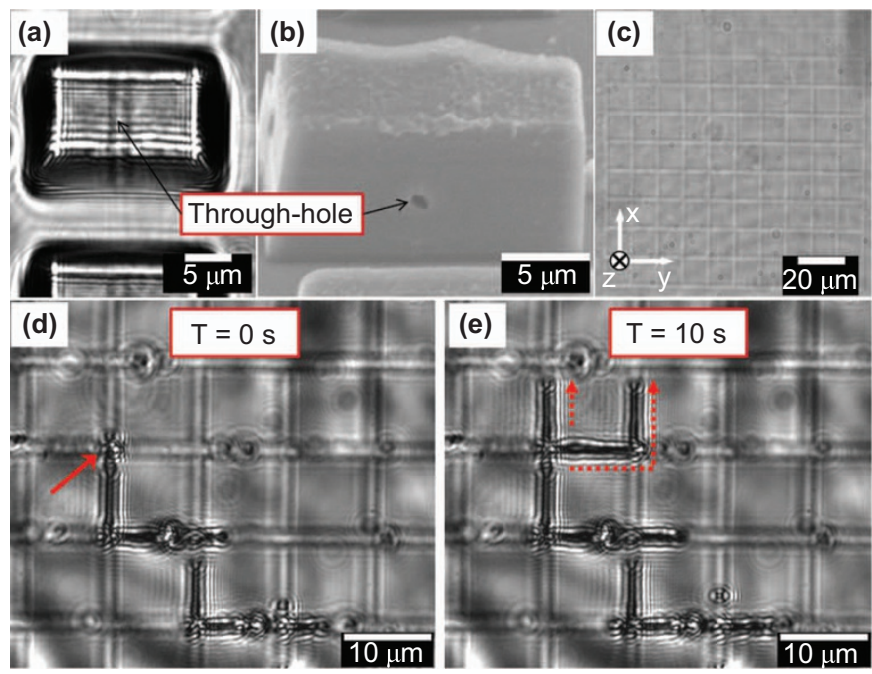

Figure 4 Two-dimensional meshed microfluidic channels inside IPL polymer fabricated by the 'TPP+FLA' method. (a) Optical microscope image (transmission mode) of a typical microfluidic channel inside a polymer cube; (b) SEM cross-section image of the microfluidic channel; (c) optical microscope image (transmission mode) of the fabricated microfluidic mesh channels; (d, e) the liquid flow inside the meshed channels at $t=0$ and $t=10 \mathrm{~s}$, respectively. The dash line shows the pathway of liquid flow through the meshed microfluidic channels. FLA, femtosecond laser ablation; SEM, scanning electron microscope; TPP, two-photon polymerization. reproducible subtractive fabrication with minimized thermal stress and collateral damages.

To demonstrate the capabilities of the 'TPP +FLA' method in micro-/nanofabrication, we fabricated two kinds of device structures. The first consists of arrays of microstructured polymer fibers with different diameters, as shown in Figure 3. The TPP process was employed to fabricate these structures with 2,1 and $0.5 \mu \mathrm{m}$ line widths, as shown in Figure 3a, c and e, respectively. The average laser power and stage scanning speed employed for TPP were $7 \mathrm{~mW}$ and $100 \mu \mathrm{m} \mathrm{s}^{-1}$, respectively. The different line widths of the fibers were achieved by controlling the number of laser scans in fabricating a single polymer fiber. Upon polymerization, the refractive index of IP-L increases to a value of 1.52 . Therefore, the polymer fibers could be used as light waveguides for integrated optics. ${ }^{21}$ Following the TPP, a subtractive FLA process was performed with an average laser power of $26 \mathrm{~mW}$, a laser exposure time of $5 \mathrm{~ms}$ per spot, and a stage scanning speed of $100 \mu \mathrm{m} \mathrm{s}^{-1}$. Periodic holes with diameters of $\sim 500 \mathrm{~nm}$ were fabricated along the polymer fibers to form Bragg grating structures in waveguides, as shown in Figure $\mathbf{3 b}$, $\mathbf{d}$ and $\mathbf{f}$. The diameter and the periodicity of the holes were tunable by adjusting the average laser power, exposure time and scanning speed. It is noteworthy that the fiber Bragg gratings, as shown in Figure 3, are difficult to be fabricated by either TPP or FLA alone.

The second type of device structures fabricated by combining both TPP and FLA is shown in Figures $\mathbf{4}$ and $\mathbf{5}$ in which meshed and spiral microfluidic channel systems are represented, respectively. In the fabrication process, polymer cubes were first fabricated by the additive process of TPP on glass substrates. Then, interconnecting microchannels were directly written within the polymer cubes via the subtractive process of FLA. The average laser power and the scanning speed employed to create the channels were $26 \mathrm{~mW}$ and $50 \mu \mathrm{m} \mathrm{s}^{-1}$, respectively. Figure $4 \mathbf{a}$ and $\mathbf{b}$ clearly shows the typical microchannels created by FLA. The diameter of the channel is about $1 \mu \mathrm{m}$ according to the scanning electron microscopy (SEM) characterization. Figure 4c shows the meshed microfluidic channel system created inside the IP-L polymer cube. Liquid flow through the meshed channels can be clearly observed in Figure $\mathbf{4 d}$ and $\mathbf{e}$, which verifies the hollow structure and the connectivity of the microfluidic channels (a video of the liquid flow is provided in the Supplementary Movie 1). Besides the 2D meshed channels, 3D spiral microchannels were also successfully fabricated inside the IP-L polymer. Figure 5a shows a schematic of 3D spiral channel inside a polymer cube. The labels of ' $A$ ' and ' $\mathrm{B}$ ' in Figure 5 refer to two points which are the highest and the lowest in the $\mathrm{Z}$ direction, respectively. The optical microscope image of the spiral channel changes as the focal plane of observation shifts from low to high, as shown in Figure $\mathbf{5 b} \mathbf{b} \mathbf{d}$, which indicates the $3 \mathrm{D}$ characteristics of the spiral microfluidic channel created inside the IP-L cube. By using the 'TPP+FLA' method, arrays of the spiral microfluidic channels with a user-defined spacing can be readily fabricated, as shown in Figure 5e. Comparing with the mainstream technique of soft lithography for fabricating microfluidic systems, the 'TPP + FLA' method holds several advantages. First, it is a mask-free process. It provides an unparalleled convenience and freedom in prototype design with a computer-aided design program without the need to fabricate replica molds as always required by the soft lithography method. Second, it is a truly 3D fabrication method in contrast to the soft lithography which is inherently limited to $2 \mathrm{D}$ processing, due to the requirement of molding. Third, it needs no sealing of microchannels which is a necessary fabrication step in the soft lithography process. 

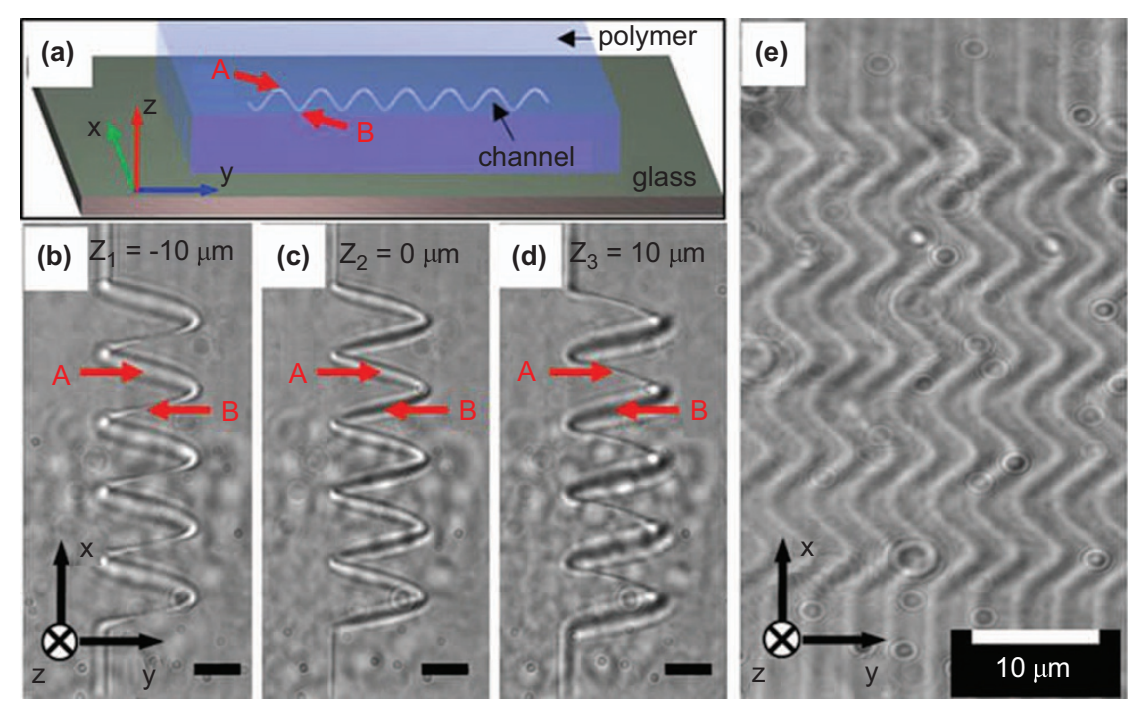

Figure 5 Three-dimensional spiral microfluidic channels inside an IP-L polymer cube fabricated by the 'TPP+FLA' method. (a) Schematic of the three-dimensional spiral microfluidic channel; (b, c, d) the X-Y cross-sectional view of a spiral channel under a transmission-mode optical microscope at different focal planes (scale bar: $10 \mu \mathrm{m}$ ). The coil diameter of the spiral channel is $20 \mu \mathrm{m}$; (e) array of spiral microfluidic channels fabricated inside a polymer cube with a coil diameter of $5 \mu \mathrm{m}$ and an interchannel spacing of $3 \mu \mathrm{m}$. FLA, femtosecond laser ablation; SEM, scanning electron microscope; TPP, two-photon polymerization.

\section{CONCLUSIONS}

In conclusion, a novel and versatile 3D micro-/nanofabrication method was developed by integrating both additive TPP and subtractive FLA processes into a single framework using fs laser direct writing techniques. The nonlinear characteristics of the fabrication processes offer a writing resolution below $200 \mathrm{~nm}$, far beyond the optical diffraction limit. In addition, this method also inherits the virtues of a sharp and clean processing edge due to the minimized thermal stress and collateral damages. The combination of both additive and subtractive fabrication processes enables the fabrication of complex 3D micro-/nanostructures which are difficult for either TPP or FLA alone. This method paves the way for the development of advanced devices such as integrated optical circuits and lab-on-a-chip devices.

\section{ACKNOWLEDGMENTS}

This research work was financially supported by National Science Foundation (CMMI 0900419 and 0758199) and National Natural Science Foundation of China (grant no. 90923039).

1 Cumpston BH, Ananthavel SP, Barlow S, Dyer DL, Ehrlich JE et al. Two-photon polymerization initiators for three-dimensional optical data storage and microfabrication. Nature 1999; 398: 51-4.

2 Seet KK, Mizeikis V, Juodkazis S, Misawa H. Spiral three-dimensional photonic crystals for telecommunications spectral range. Appl Phys A-Mater 2006; 82: 683-8.

3 Reinhardt C, Kiyan R, Passinger S, Stepanov AL, Ostendorf A et al. Rapid laser prototyping of plasmonic components. Appl Phys A-Mater 2007; 89: 321-5.

4 Reinhardt C, Passinger S, Chichkov BN, Marquart C, Radko IP et al. Laser-fabricated dielectric optical components for surface plasmon polaritons. Opt Lett 2006; 31: 1307-9.

5 Klein F, Striebel T, Fischer J, Jiang ZX, Franz CM et al. Elastic fully three-dimensional microstructure scaffolds for cell force measurements. Adv Mater 2010; 22: 868-71.
6 Cunningham LP, Veilleux MP, Campagnola PJ. Freeform multiphoton excited microfabrication for biological applications using a rapid prototyping CAD-based approach. Opt Express 2006; 14: 8613-21.

7 Maruo S, Ikuta K, Korogi H. Submicron manipulation tools driven by light in a liquid. Appl Phys Lett 2003; 82: 133-5.

8 Maruo S, Ikuta K, Korogi H. Force-controllable, optically driven micromachines fabricated by single-step two-photon micro stereolithography. J Microelectromech S 2003; 12: 533-9.

9 Maruo S, Inoue H. Optically driven micropump produced by three-dimensional twophoton microfabrication. Appl Phys Lett 2006; 89: 144101.

10 Galajda P, Ormos P. Rotors produced and driven in laser tweezers with reversed direction of rotation. Appl Phys Lett 2002; 80: 4653-5.

11 Galajda P, Ormos P. Complex micromachines produced and driven by light. Appl Phys Lett 2001; 78: 249-51.

12 Maruo S, Fourkas JT. Recent progress in multiphoton microfabrication. Laser Photon Rev 2008; 2: 100-11.

13 Zhang YL, Chen QD, Xia H, Sun HB. Designable 3D nanofabrication by femtosecond laser direct writing. Nano Today 2010; 5: 435-48.

14 Gattass RR, Mazur E. Femtosecond laser micromachining in transparent materials. Nat Photon 2008; 2: 219-25.

15 Baldacchini T. Novel techniques for the fabrication of two- and three-dimensional microstructures. PhD thesis, Boston College, Boston, MA, 2004. http:// www. proquest.com/

16 Glezer EN, Milosavljevic M, Huang L, Finlay RJ, Her TH et al. Three-dimensional optical storage inside transparent materials. Opt Lett 1996; 21: 2023-5.

17 Marshall GD, Ams M, Withford MJ. Direct laser written waveguide-Bragg gratings in bulk fused silica. Opt Lett 2006; 31: 2690-1.

18 Shen N, Datta D, Schaffer CB, LeDuc P, Ingber DE et al. Ablation of cytoskeletal filaments and mitochondria in live cells using a femtosecond laser nanoscissor. Mech Chem Biosyst 2005; 2: 17-25.

19 Boyd RW. Nonlinear Optics. 3rd ed. Amsterdam/Boston: Academic Press; 2008.

20 Goldman JR, Prybyla JA. Ultrafast dynamics of laser-excited electron distributions in silicon. Phys Rev Lett 1994; 72: 1364-7.

21 Zappe HP. Fundamentals of Micro-optics. Cambridge/New York: Cambridge University Press; 2010.

This work is licensed under a Creative Commons Attribution-NonCommercial-NoDerivative Works 3.0 Unported License. To view a copy of this license, visit http:// creativecommons.org/licenses/by-nc-nd/3.0 